\title{
Antisense repression in Cryptococcus neoformans as a laboratory tool and potential antifungal strategy
}

\author{
Jenifer M. Gorlach, Henry C. McDade, John R. Perfect and Gary M. Cox
}

Author for correspondence: Gary M. Cox. Tel: +1 919681 5055. Fax: +1 919684 8902. e-mail: gary.cox@duke.edu

Departments of Medicine and Microbiology, Duke University Medical Center, Durham, NC, USA

\begin{abstract}
Antisense repression was used as a method to alter gene function in the human-pathogenic fungus Cryptococcus neoformans. The calcineurin A gene (CNA1) and the laccase gene (LAC1) were targeted since disruption of these loci results in phenotypes that are easy to screen (temperature sensitivity and lack of melanin, respectively). Serotype $D$ yeasts were transformed with a plasmid containing the CNA1 CDNA in an antisense orientation under the control of the inducible GAL7 promoter, and serotype A yeasts were transformed with a plasmid containing the LAC1 CDNA in an antisense orientation under the control of the constitutive actin promoter. The calcineurin transformants demonstrated a temperature-sensitive phenotype only when grown on galactose, and the laccase transformants had decreased melanin production. Northern blot analysis of the calcineurin antisense transformants confirmed that the inducible phenotype was associated with a decrease in the native CNA1 transcript levels. Furthermore, it was possible to modestly impair growth of $\mathrm{C}$. neoformans at $37^{\circ} \mathrm{C}$ by using a $30 \mathrm{bp}$ antisense oligonucleotide targeting CNA1. Antisense repression is now available as a tool for molecular studies in this organism, and may be applicable to other human-pathogenic fungi that have less amenable genetic systems.
\end{abstract}

Keywords: calcineurin, antisense oligonucleotide, yeast, fungus, pathogenesis

\section{INTRODUCTION}

Cryptococcus neoformans is one of the most significant pathogenic fungi to infect immunocompromised humans. The treatment of this infection, and of all fungal infections in general, is associated with high morbidity and mortality (Casadevall \& Perfect, 1998). The hope is that basic research on the pathogenesis of fungal infections will identify targets for the development of improved treatment strategies. In this regard, C. neoformans has proven to be an excellent model system for molecular pathogenesis studies (Perfect, 1996).

Antisense repression is a means to alter gene function that works at the level of mRNA rather than at the level of the genomic locus (Inouye, 1988; Kuss \& Cotter, 1999; Persidis, 1999). Antisense repression starts by having single-stranded RNA that is complementary to

Abbreviations: RACE, rapid amplification of CDNA ends; 5-FOA, 5fluoroorotic acid. the target mRNA. The antisense RNA binds to the target mRNA to form an RNA-RNA duplex, and the target mRNA is inactivated, possibly as a result of degradation or inability to access the translational machinery (Liu \& Carmichael, 1994; Kumar \& Carmichael, 1998).

Antisense repression has several features that can be complementary to the standard methods of gene inactivation using targeted disruption. In conventional gene disruption strategies, especially in an organism like C. neoformans whose genome has many introns, the genomic locus must be cloned. Antisense repression does not require the isolation of the genomic locus, and gene inactivation can be accomplished by using relatively short pieces of coding sequence rather than entire genes. This allows for the use of techniques such as RACE (rapid amplification of cDNA ends) to quickly make constructs for mutagenesis. Another advantage is that antisense repression does not require homologous integration, and it may be especially useful for other fungal organisms in which rates of homologous re- 
combination are very low. Another potential use for antisense repression is the simultaneous inactivation of multiple loci by using a single antisense molecule that targets a sequence common to all of the target mRNAs. For example, a single antisense transcript affected the expression of all three discoidin I genes in Dictyostelium, a feat that otherwise would have required three separate rounds of targeted disruption (Crowley et al., 1985). In addition, a single antisense oligonucleotide targeted against the multicopy gene encoding gp90 of Trypanosoma cruzi was able to inhibit function in vivo (Malaga \& Yoshida, 2001). Genes essential for vegetative growth can be identified with antisense repression (Liu et al., 1992; Tentler et al., 1997), and this was also recently demonstrated in Candida albicans (DeBacker et al., 2001). Proving that genes are essential to an organism is extremely complicated since they cannot be easily identified using conventional gene knockout strategies. However, genes essential to the organism may be ideal targets for the development of new antifungal drugs since inhibition of function would confer fungicidal rather than fungistatic activities.

Antisense repression is not only useful as a laboratory tool, but has also been used in vivo as an antibiotic and to alter host gene function. There is currently an FDAapproved medication consisting of antisense oligonucleotides for the treatment of cytomegalovirus (CMV) retinitis (Perry \& Balfour, 1999), and there are clinical trials in humans that are using antisense oligonucleotides to treat leukaemia and other malignancies (Kuss \& Cotter, 1999). Furthermore, investigators have injected antisense oligonucleotides into experimental animals to alter gene expression of both the host (McKinley et al., 2000) and the pathogen (Chakraborty et al., 1999).

In this paper we describe the adaptation of antisense repression to the study of a human-pathogenic fungus, and the potential use of antisense oligonucleotides as antifungals. The availability of this technique should facilitate future molecular studies using Cryptococcus neoformans, and we also propose that antisense repression can be applied to other human-pathogenic fungi. Antisense repression has already proven itself to be valuable in the diploid Candida albicans, where a single transformation event has resulted in altered gene function (DeBacker et al., 2001). Antisense repression may also be valuable in molecular studies of other fungi such as Coccidioides immitis and Histoplasma capsulatum where genetic manipulations are much more technically difficult.

\section{METHODS}

Cryptococcus neoformans strains and media. JEC43 (ura5) and JEC50 (ade2) were derived from JEC21 (serotype D, mating type $\alpha$ ) and were a generous gift from Jeff Edman. MCC1 (cna1:: ADE2) was derived from JEC50 (Cruz et al., 2000) and was a generous gift from M. Cristina Cruz and Joseph Heitman. HMC6 (lac1::URA5) was derived from JEC21 (Rosas et al., 2000). Strain H99 is a wild-type, serotype $\mathrm{A}$, mating type $\alpha$ strain, and $\mathrm{H} 99 \mathrm{R}$ is a spontaneous ura5 auxotroph that resulted from plating $\mathrm{H} 99$ on 5-FOA medium.

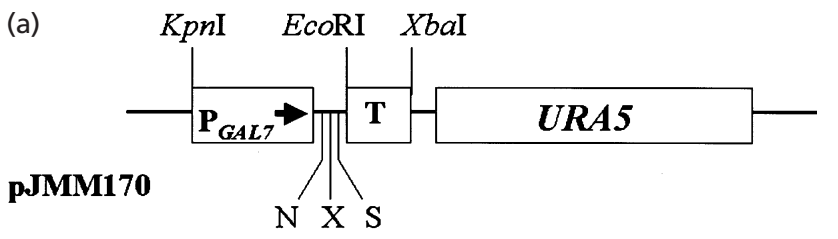

(b)

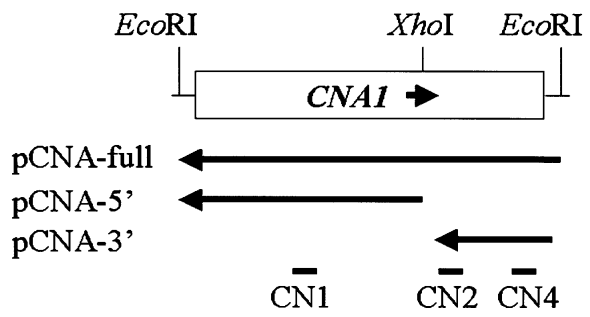

Fig. 1. (a) Map of the relevant sites in the antisense vector pJMM170. $\mathrm{P}_{\text {GAL71 }}$ GAL7 promoter; $\mathrm{T}$, TRP1 terminator sequence; N, Notl; X, Xhol; S, Stul. (b) Partial map of the cloned CNA1 CDNA that was used in the antisense vectors. The leftpointing arrows represent the portions of the CDNA that were cloned in an antisense orientation into pJMM170 for each antisense vector. The approximate locations of CNA1 that were used to design antisense oligonucleotides CN1, CN2 and CN4 are also noted.

(a)

pJMM180

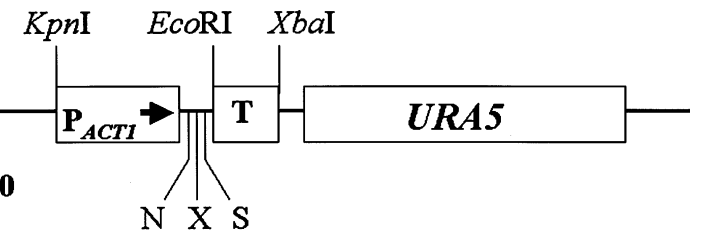

(b)

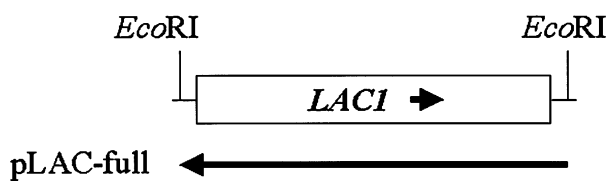

Fig. 2. (a) Map of the relevant sites in the antisense vector pJMM180. $\mathrm{P}_{\mathrm{ACT} 1}, A C T 1$ promoter; $\mathrm{T}$, TRP1 terminator sequence; N, Notl; X, Xhol; S, Stul. (b) The full-length LAC1 cDNA was amplified and cloned into a vector, from which it was digested using EcoRI and subcloned into pJMM180 to result in pLAC-full.

All strains were stored in glycerol stocks at $-80{ }^{\circ} \mathrm{C}$, and were maintained on YEPD agar $(1 \%$ yeast extract, $2 \%$ peptone, $2 \%$ dextrose [glucose] and $1.8 \%$ agar). Induction of antisense vector was done on YEPGal ( $1 \%$ yeast extract, $2 \%$ peptone, $2 \%$ galactose). Minimal medium plates were made using $0.67 \%$ yeast nitrogen base without amino acids, $2 \%$ glucose and $1.8 \%$ agar. Dopamine medium was made as described by Rosas et al. (2000).

Antisense vectors. The antisense expression vectors (Figs 1 and 2) were constructed by first amplifying a $310 \mathrm{bp}$ fragment containing the terminator sequence and stop codon from the C. neoformans strain H99 TRP1 (Perfect et al., 1992) using primers to introduce EcoRI and XbaI sites at the $5^{\prime}$ and $3^{\prime}$ ends, respectively (CTGAATTCTGAAGGCGGTAAGGGG- 
TTAA and TGTCTAGAGCGACAGAAGAGAAATGGTGA; enzyme sites underlined). The amplicon was digested with EcoRI and $\mathrm{XbaI}$, and cloned into pBluescript SK - (Stratagene). The resulting plasmid was digested with $X b a \mathrm{I}$ and SacII, and a fill-in reaction using Taq polymerase was performed. Into this blunt site was cloned a $2012 \mathrm{bp}$ fragment containing the C. neoformans UR A5 (Edman \& Kwon-Chung, 1990), which was digested from pCnTEL (Edman, 1992) using $C l a \mathrm{I}$ and EcoRI, and blunt-ended using Taq polymerase. A $624 \mathrm{bp}$ fragment containing the promoter and ATG start site from the C. neoformans strain JEC21 GAL7 (Wickes \& Edman, 1995) was amplified using primers to introduce a $K p n \mathrm{I}$ site at the $5^{\prime}$ end and NotI, XhoI, StuI and EcoRI sites at the $3^{\prime}$ end (GAGGTACCATCACACATTTGTTCCGCCGGT and GAGAATTCAGGCCTCTCGAGAGGCGGCCGCCATTCTCAAGAGGGGATTGAGCGCTGATAA). The amplicon was digested with KpnI and EcoRI and ligated into the plasmid containing URA5 and the TRP1 terminator sequence to result in pJMM170 (Fig. 1a). An 860 bp fragment containing the promoter and ATG start site from the C. neoformans strain H99 actin gene (Cox et al., 1995) was amplified using primers to introduce the same sites on the ends as above (ATGGTACCACTCGCTATTGTCCAGGCT and GA GAATTCAGGCCTCTCGAGAGGCGGCCGCCATAGACATGTTGGGCGAGT). The amplicon was digested with $K p n I$ and EcoRI and ligated into the plasmid containing URA5 and the TRP1 terminator sequence to result in pJMM180 (Fig. 2a). All ligations were confirmed with sequencing.

The full-length, 1920 bp CNA1 cDNA from C. neoformans strain B3501 was amplified using a pooled cDNA library as template. The amplicon was subcloned to plasmid and sequenced to confirm its identity. The cloned CNA1 cDNA was digested from the plasmid (pCR2.1, Invitrogen) using EcoRI, and subcloned to the EcoRI site of pJMM170. Several subclones were digested with XhoI to identify a plasmid where the CNA1 cDNA had been cloned into pJMM170 in an antisense orientation, and this plasmid was designated pCNAfull (Fig. 1b). A partial CNA1 cDNA fragment containing the first $1330 \mathrm{bp}$ extending from the ATG start codon to a XhoI site was digested from the cloned CNA1 cDNA using XhoI and EcoRI, and subcloned to PJMM170 with the designation pCNA-5' (Fig. 1b). Another partial cDNA fragment containing the last $570 \mathrm{bp}$ of CNA1 was amplified to introduce $X$ XoI and EcoRI sites into the $3^{\prime}$ and $5^{\prime}$ ends, respectively, and the amplicon was ligated into pJMM170 with the designation pCNA-3' (Fig. 1b). The full-length, 1870 bp LAC1 cDNA from strain B3501 was amplified using a pooled cDNA library as template, and subcloned to plasmid for sequencing. The cloned LAC1 cDNA was digested from the plasmid (pCR2.1, Invitrogen) using EcoRI, and subcloned to the EcoRI site of pJMM180 (Fig. 2b). Several plasmids were screened using PCR to identify one containing the LAC1 cDNA in an antisense orientation behind the actin promoter (pLAC-full, Fig. 2b).

Transformation of strains JEC43 and H99R was performed using biolistic DNA delivery of intact plasmid as described by Davidson et al. (2000). Transformants were selected on minimal medium at $30{ }^{\circ} \mathrm{C}$.

Antisense oligonucleotides having phosphorothioate substitutions were obtained unpurified from the manufacturer (Operon) with the following sequences: CN1, ataCCACATCTTGAGCGACCACAAATAaagC; CN2, gggATGGGGCGTACAATTGAATTGTCggaT; CN4, cgcCAAGCAGTGAGTGGCGGACCTCCAatgG (phosphorothioate-substituted nucleotides in lower case; approximate locations of each oligonucleotide within CNA1 shown in Fig. 1b). Oligonucleotides were suspended in TE buffer, aliquoted and frozen at $-20{ }^{\circ} \mathrm{C}$. Yeast experiments using oligonucleotides were done by diluting OligofectAMINE reagent (Life Technologies) 1:5 with RPMI ( $\mathrm{pH} 7 \cdot 0$ ) medium. Ten microlitres of the diluted reagent was mixed with various concentrations of oligonucleotides and left to incubate at room temperature for $20 \mathrm{~min}$. The oligonucleotide/OligofectAMINE mixtures were then placed into 96-well plates, and $10 \mu \mathrm{l}$ of a suspension of $10^{6}$ c.f.u. of strain $\mathrm{H} 99$ cryptococci ml ${ }^{-1}$ was added to each well. The total volume of each well was adjusted to $100 \mu \mathrm{l}$ with RPMI, and the plates were incubated for $24 \mathrm{~h}$ at $37^{\circ} \mathrm{C}$ with shaking. Growth was assessed using duplicate quantitative cultures. Each experiment was repeated three separate times and the data were averaged.

\section{RESULTS AND DISCUSSION}

\section{Transformation with the antisense construct}

In order to adapt antisense repression for use in $C$. neoformans, we targeted the CNA1 and LAC1 genes. Previous studies have shown that $c n a 1$ and lac1 mutants of C. neoformans generated via targeted gene disruption are unable to grow at $37^{\circ} \mathrm{C}$ (Cruz et al., 2000; Odom et al., 1997) and lack melanin (Rosas et al., 2000; Salas et al., 1996), respectively. Both a full-length CNA1 cDNA and partial cDNA fragments were placed in an antisense orientation $\left(3^{\prime}\right.$ to $\left.5^{\prime}\right)$ in the antisense vector pJMM170, where transcription is under the control of the inducible GAL7 promoter (Fig. 1). The full-length LAC1 cDNA was placed in an antisense orientation in pJMM180, where transcription is controlled by the actin promoter (Fig. 2). The antisense constructs were used to transform yeast so as to integrate the vector into the genome in a random, ectopic fashion. Southern blots of selected pCNA-full and pCNA-5' transformants demonstrated that the native CNA1 was intact, and that the antisense construct appeared to integrate randomly with multiple, tandem repeats (data not shown).

\section{Demonstration of antisense effect}

The calcineurin transformants were screened for phenotype on glucose- and galactose-containing media at both $30^{\circ} \mathrm{C}$ and $37^{\circ} \mathrm{C}$ (Fig. 3). A cna1 strain (MCC1), wildtype and an 'empty vector' transformant (transformed with pJMM170 alone) were used as controls. The cna1 strain demonstrated the expected phenotype of no growth at $37^{\circ} \mathrm{C}$ on both YEPD and YEPGal, whereas the empty vector control was able to grow on both media at both temperatures (Fig. 3). Ten independent transformants containing the full-length CNA1 antisense construct (pCNA-full) were able to grow on both YEPD and YEPGal at $30^{\circ} \mathrm{C}$ and YEPD at $37^{\circ} \mathrm{C}$ (data for five of these are shown in Fig. 3). However, the antisense transformants were not viable under conditions of induction (YEPGal) at $37^{\circ} \mathrm{C}$ (Fig. 3).

In order to see if partial cDNA fragments could be used in antisense repression, transformants were made using the constructs pCNA-5' and pCNA-3' (Fig. 1b). Six transformants resulting from both plasmids were found 

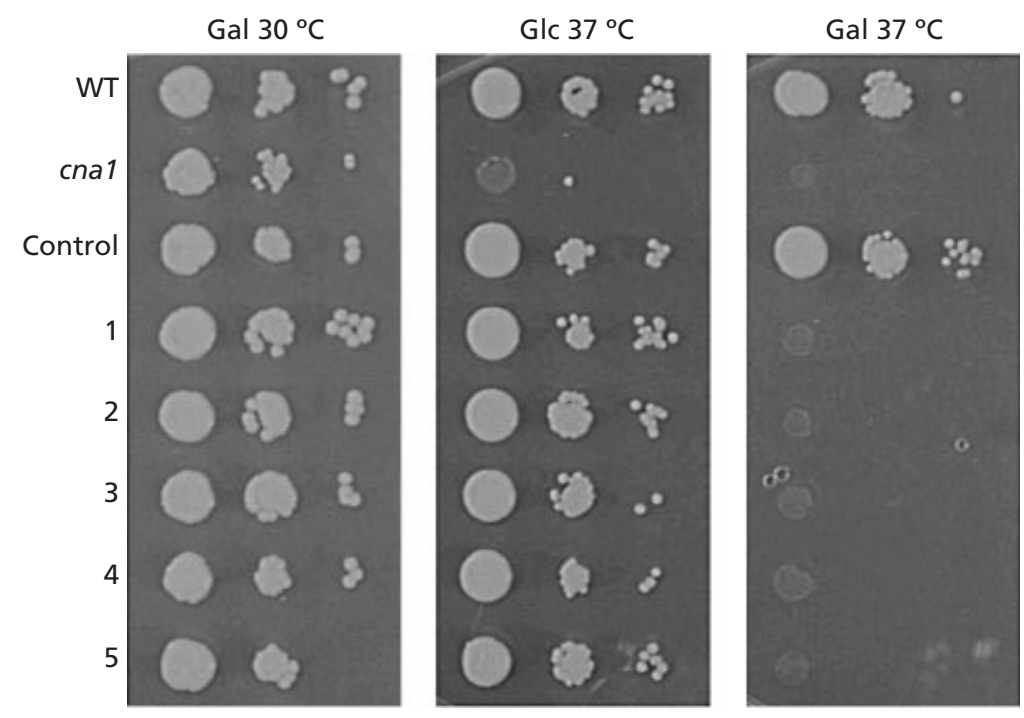
Fig. 3. Transformants containing the CNA1 antisense vector displayed an inducible phenotype identical to the cna1 knockout strain. Serial 1:10 dilutions of each strain were plated onto YEPGal (Gal, galactose) or YEPD (Glc, glucose) and grown at $30^{\circ} \mathrm{C}$ and $37^{\circ} \mathrm{C}$. WT, wild-type strain JEC21; cna1, calcineurin A mutant generated by targeted gene disruption (strain MCC1); control, 'empty vector' control strain generated using pJMM170; 1-5, five independent strains generated with the CNA1 antisense construct pCNA-full.

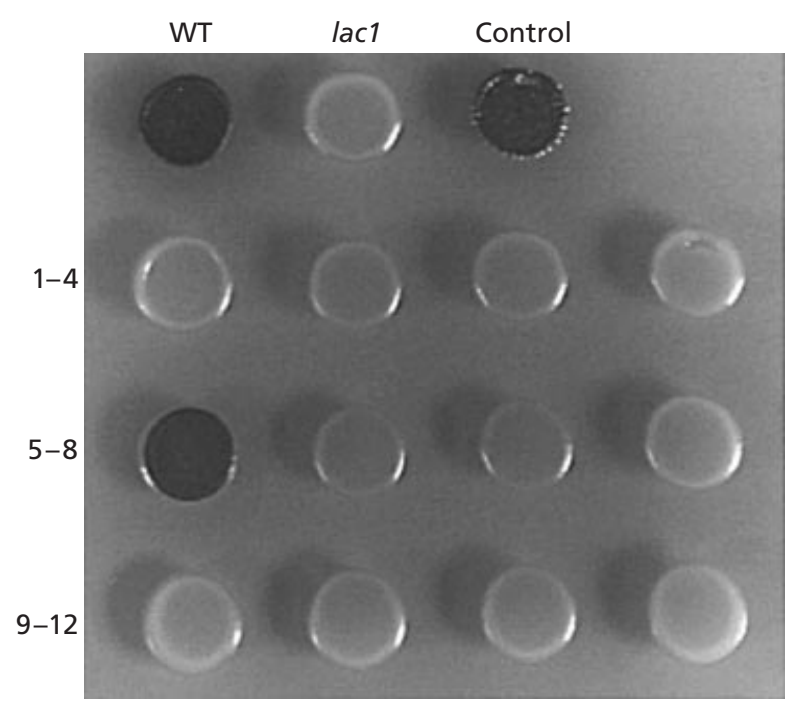

Fig. 4. Transformants containing the antisense vector targeting LAC1 displayed an albino phenotype identical to the lac1 knockout strain. All strains were spotted onto dopamine agar, and 11 of the 12 strains generated with the pLAC-full construct (strains 1-4, 5-8, 9-12) were identical to strain HMC6 (lac1). WT, wild-type strain H99; control, 'empty vector' control strain generated using pJMM180.

to have phenotypes identical to transformants resulting from the construct containing the full-length cDNA (growth on all conditions except on YEPGal at $37^{\circ} \mathrm{C}$; data not shown).

The laccase antisense construct was used to transform the serotype A strain H99R. Twelve randomly selected transformants were screened on dopamine agar with H99 (wild-type), a lac1 strain (HCM6) and an 'empty vector' transformant as controls (Fig. 4). Eleven of the twelve antisense transformants had the albino phenotype of the lac1 strain, and one appeared to make melanin near wild-type levels (Fig. 4).

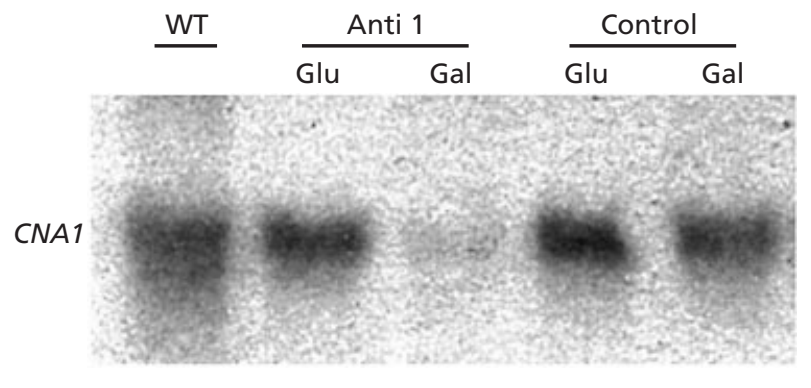

Fig. 5. Northern blot demonstrating decreased levels of native CNA1 transcript in an antisense strain generated with pCNA-5' (Anti 1 ) only when the cells are grown with galactose (Gal) and not with glucose (Glu). Total RNA from JEC21 (WT) and an 'empty vector' strain (Control) were included. The blot was stripped and reprobed with actin, which confirmed equivalent loading of RNA into each well (data not shown).

\section{Confirmation of phenotype with Northern analysis}

Northern analysis was performed using a transformant containing the pCNA-5' construct. To avoid crossreactivity between the antisense and native transcripts, the blot was probed with a labelled CNA1 fragment from the $3^{\prime}$ end of the gene that was not included in the pCNA-5' construct. The blot established that the amount of CNA1 transcript was markedly decreased in the transformant when grown under the conditions of galactose induction (Fig. 5). An empty vector control strain demonstrated no significant differences in CNA1 expression in yeast grown on glucose as compared to galactose medium (Fig. 5).

\section{Use of antisense oligonucleotides}

Three 30 bp antisense oligonucleotides that are complementary to various portions of the CNA1 coding sequence were synthesized with the terminal three residues of each oligonucleotide having a phosphoro- 
Table 1. Effects of oligonucleotides on the growth of strain H99

\begin{tabular}{|c|c|c|c|c|c|c|c|}
\hline & \multicolumn{7}{|c|}{ Oligonucleotide: } \\
\hline & \multirow[t]{2}{*}{ None } & \multicolumn{2}{|c|}{ T7 (control) } & \multirow{2}{*}{$\begin{array}{c}\text { CN1 } \\
40 \mu \mathrm{g} \mathrm{ml}^{-1}\end{array}$} & \multirow{2}{*}{$\begin{array}{c}\mathrm{CN} 2 \\
40 \mu \mathrm{g} \mathrm{ml}^{-1}\end{array}$} & \multicolumn{2}{|c|}{$\mathrm{CN} 4$} \\
\hline & & $40 \mu \mathrm{g} \mathrm{ml}^{-1}$ & $400 \mu \mathrm{g} \mathrm{ml}^{-1}$ & & & $40 \mu \mathrm{g} \mathrm{ml}^{-1}$ & $400 \mu \mathrm{g} \mathrm{ml}^{-1}$ \\
\hline \multicolumn{8}{|l|}{$37^{\circ} \mathrm{C}$} \\
\hline No. of yeast" (SD) & $80(8)$ & $82(8)$ & $26(11)$ & $66(10)$ & $86(7)$ & $40(4)$ & $23(14)$ \\
\hline$P$ valuet & $0 \cdot 46$ & & $0 \cdot 01$ & $0 \cdot 10$ & $0 \cdot 69$ & $0 \cdot 02$ & $0 \cdot 01$ \\
\hline \multicolumn{8}{|l|}{$30{ }^{\circ} \mathrm{C}$} \\
\hline No. of yeast* (SD) & $478(23)$ & $394(69)$ & ND & $457(87)$ & $408(57)$ & $412(37)$ & ND \\
\hline$P$ valuet & $0 \cdot 09$ & & & $0 \cdot 10$ & $0 \cdot 12$ & $0 \cdot 12$ & \\
\hline
\end{tabular}

* Number of yeasts counted per plate of standard dilution $\left(10^{-5}\right)$ at $24 \mathrm{~h}$ with standard deviation in parentheses. Values represent the mean of three separate experiments with two plates counted per experiment. The T7 primer was used as a control for the three CNA1 antisense oligonucleotides CN1, CN2 and CN4.

† Student's $t$-test compared to the $\mathrm{T} 7 / 40 \mu \mathrm{g} \mathrm{ml} \mathrm{g}^{-1}$ group.

thioate-substituted sugar in order to provide resistance to nuclease degradation (Fig. 1b). The choices of the sequences targeted by the oligonucleotides were based on low levels of predicted secondary structure in the CNA1 mRNA, and the chosen sequences were conserved in both the H99 and B3501 strains. The oligonucleotides were mixed with wild-type C. neoformans strain $\mathrm{H} 99$ at different concentrations, and grown in RPMI medium at $37^{\circ} \mathrm{C}$. A T7 primer was used as a control oligonucleotide. None of the calcineurin antisense oligonucleotides appeared to have significant effects on growth at a concentration of $4 \mu \mathrm{g} \mathrm{ml}^{-1}$ as compared to both the no-oligonucleotide control and the T7 control (data not shown). At the $40 \mu \mathrm{g} \mathrm{ml}^{-1}$ concentration, only the CN4 oligonucleotide had a significant impact on high-temperature growth (Table 1), although the decrease in cell number compared to the two control groups was relatively modest. The growth rates were not affected by the same concentration of the $\mathrm{CN} 4$ oligonucleotide when the yeasts were grown at $30^{\circ} \mathrm{C}$ (Table 1). Higher concentrations of oligonucleotides were used, but growth rates for both the T7 and calcineurin antisense groups were similarly decreased in the $400 \mu \mathrm{g} \mathrm{ml}^{-1}$ assays (Table 1 ).

\section{Conclusions}

This is the first adaptation of antisense repression to the study of Cryptococcus neoformans. We constructed antisense vectors containing a multicloning site that allows for cDNA fragments from either standard libraries or RACE protocols to be directly ligated into the vector in an antisense orientation behind the promoter. One of the vectors has an inducible GAL7 promoter for use in serotype $\mathrm{D}$ strains, and the other has an actin promoter for constitutive expression in serotype A strains. Transformation using the antisense vectors and biolistic DNA delivery results in integration into the genome, often with multimeric repeats. Both the in- tegration into the genome and the repeats work to the advantage of antisense repression since the antisense transcripts are made in high copy number in the nucleus so as to have the best chance of associating with the target mRNA.

Using the antisense vector to target CNA1 in a serotype D strain and LAC1 in a serotype A strain, we were able to demonstrate that the function of these genes could be altered by antisense repression. This was demonstrated by the fact that transformants containing the antisense vector had the expected phenotype of the corresponding knockout mutants. In the case of the calcineurin antisense mutants, the antisense phenotype was only observed under the conditions of induction of the antisense transcript. Furthermore, the level of CNA1 mRNA in an antisense transformant was markedly decreased only when the cells were grown under the conditions of induction, suggesting that the native mRNA was destroyed by the antisense transcripts. The use of an inducible promoter not only helps with demonstrating that antisense repression is responsible for the observed phenotypes, but also provides for the screening of essential genes. Antisense repression seems broadly applicable for use in C. neoformans since we have demonstrated success by targeting different genes in both of the main serotypes used in molecular studies of this yeast.

We were able to target CNA1 using antisense repression by using both whole and partial cDNA sequences as template for the antisense transcripts. Because one potential use of antisense repression is to screen cDNA libraries for phenotypes of interest, it was important to verify that partial cDNA sequences, especially from the $3^{\prime}$ portion of the gene, can be used in antisense repression. It is difficult to predict how small an antisense transcript can be and still disrupt gene function since the efficiency of binding to the target mRNA is dependent on multiple different factors including copy 
number of the native mRNA and secondary structure. It is also likely that genes differ widely in the number of mRNA copies that are necessary for wild-type function. Antisense repression probably cannot completely abolish all native mRNAs, and some genes may have wildtype function with only very few copies of mRNA present, whereas other genes may require many copies. It appears that the C. neoformans CNA1 may be an example of the latter possibility. The Northern blots of transformants grown under the conditions of induction show that the levels of the native CNA1 mRNA were very low, but still detectable. However, the transformants were still unable to grow at high temperatures, suggesting that high levels of message are needed for wild-type expression of this particular phenotype.

In order to test whether very small pieces of DNA could also be used in antisense repression in C. neoformans, we used 30 bp oligonucleotides complementary to various parts of CNA1. We used sequences that were predicted to have low secondary structure so as to select for parts of the target mRNA that are more likely to bind the antisense oligonucleotide. The sugars of the terminal three nucleotides had phosphorothioate substitutions in order to increase resistance to nucleases, and thus increase stability within the cell. Only one of the three oligonucleotides appeared to have any significant effect on growth at $37^{\circ} \mathrm{C}$, and the associated growth defect was relatively modest. There did appear to be non-specific toxicities associated with high concentrations of oligonucleotides, as demonstrated by the yeast exposed to $400 \mu \mathrm{g} \mathrm{ml}^{-1}$ of the T7 oligonucleotide. However, the growth defect at $37^{\circ} \mathrm{C}$ associated with the $\mathrm{CN} 4$ oligonucleotide does not appear to be a nonspecific toxicity since yeast grown with the same concentration of the $\mathrm{CN} 4$ oligonucleotide did not have a growth defect at $30^{\circ} \mathrm{C}$. The fact that the $\mathrm{CN} 1$ and $\mathrm{CN} 2$ oligonucleotides did not appear to affect growth as compared to both the T7 and no-oligonucleotide controls also supports the absence of non-specific toxicities at the $40 \mu \mathrm{g} \mathrm{ml} \mathrm{g}^{-1}$ concentration. Therefore, we have demonstrated that a biological effect on growth can be achieved in this pathogenic yeast with antisense oligonucleotides.

There are two possible reasons why the antisense effect using the $\mathrm{CN} 4$ oligonucleotide was so much less pronounced than when using the integrated antisense vectors. The much smaller sizes of the oligonucleotides as compared to the antisense transcripts are likely to result in less effective targeting of the native mRNA. Furthermore, the introduced antisense oligonucleotides need to be taken up by the yeasts, whereas the antisense transcripts are produced in high numbers within the nucleus. We used the OligofectAMINE reagent with the oligonucleotides to form transformation complexes in an effort to increase delivery to the yeasts since this has apparently worked with a variety of cultured eukaryotic cells (unpublished data provided by the manufacturer). However, it seems clear that in order to optimize antisense oligonucleotides for use as antifungal agents, there will have to be technological advances in the delivery of oligonucleotides to yeast. There will also have to be careful mapping of selected genes in order to identify short sequences that can be targeted with high efficiency. Ideally, the targeted sequences would be conserved across a wide range of fungi so that the oligonucleotide would have a broad spectrum of action. It is possible that antisense oligonucleotides may be useful as adjuncts to standard antifungals in the treatment of infections.

\section{ACKNOWLEDGEMENTS}

This work was done as part of the Duke University Mycology Research Unit (DUMRU), and was supported by Public Health Service grants AI01334 (G.M.C.) and AI28388 (J.R.P.) from the National Institute of Allergy and Infectious Diseases. G.M.C. was a recipient of the Burroughs Wellcome Fund New Investigator Award in Molecular Pathogenic Mycology. We would like to acknowledge Joseph Heitman for his support and helpful suggestions, and Garrett Heinrich for technical assistance. Portions of this work were presented at the Fourth International Conference on Cryptococcus and Cryptococcosis, September 1999.

\section{REFERENCES}

Casadevall, A. \& Perfect, J. R. (1998). Cryptococcus neoformans. Washington, DC: American Society for Microbiology.

Chakraborty, R., Dasgupta, D., Adhya, S. \& Basu, M. K. (1999). Cationic liposome-encapsulated antisense oligonucleotide mediates efficient killing of intracellular Leishmania. Biochem J 340, 393-396.

Cox, G. M., Rude, T. H., Dykstra, C. C. \& Perfect, J. R. (1995). The actin gene from Cryptococcus neoformans: structure and phylogenetic analysis. J Med Vet Mycol 33, 261-266.

Crowley, T. E., Nellen, W., Gomer, R. H. \& Firtel, R. A. (1985). Phenocopy of discoidin I-minus mutants by antisense transformation in Dictyostelium. Cell 43, 633-641.

Cruz, M. C., Sia, R. A. L., Olson, M., Cox, G. M. \& Heitman, J. (2000). On the roles of calcineurin in physiology and virulence in serotype D compared to serotype A strains of Cryptococcus neoformans. Infect Immun 68, 982-985.

Davidson, R. C., Cruz, M. C., Sia, R. A., Allen, B., Alspaugh, J. A. \& Heitman, J. (2000). Gene disruption by biolistic transformation in serotype D strains of Cryptococcus neoformans. Fungal Genet Biol 29, 38-48.

DeBacker, M. D., Nelissen, B., Logghe, M. \& 10 other authors (2001). An antisense-based functional genomics approach for identification of genes critical for growth of Candida albicans. Nat Biotechnol 19, 235-241.

Edman, J. C. (1992). Isolation of telomerelike sequences from Cryptococcus neoformans and their use in high-efficiency transformation. Mol Cell Biol 12, 2777-2783.

Edman, J. C. \& Kwon-Chung, K. J. (1990). Isolation of the URA5 gene from Cryptococcus neoformans var. neoformans and its use as a selective marker for transformation. Mol Cell Biol 10, 4538-4544.

Inouye, M. (1988). Antisense RNA : its functions and applications in gene regulation. Gene 72, 25-34.

Kumar, M. \& Carmichael, G. G. (1998). Antisense RNA: function and fate of duplex RNA in cells of higher eukaryotes. Microbiol Mol Biol Rev 62, 1415-1434. 
Kuss, B. \& Cotter, F. (1999). Antisense-time to shoot the messenger. Ann Oncol 10, 495-503.

Liu, Z. \& Carmichael, G. G. (1994). Nuclear antisense RNA. Mol Biotechnol 2, 107-118.

Liu, T., Williams, J. G. \& Clarke, M. (1992). Inducible expression of calmodulin antisense RNA in Dictyostelium cells inhibits the completion of cytokinesis. Mol Biol Cell 3, 1403-1413.

Malaga, S. \& Yoshida, N. (2001). Targeted reduction in expression of Trypanosoma cruzi surface glycoprotein gp90 increases parasite infectivity. Infect Immun 69, 353-359.

McKinley, M. J., Guzzo-Pernell, N. \& Sinnayah, P. (2000). Antisense oligonucleotide inhibition of angiotensinogen in the brains of rats and sheep. Methods 22, 219-225.

Odom, A., Muir, S., Lim, E., Toffaletti, D. L., Perfect, J. R. \& Heitman, J. (1997). Calcineurin is required for virulence of Cryptococcus neoformans. EMBO J 16, 2576-2589.

Perfect, J. R. (1996). Fungal virulence genes as targets for antifungal chemotherapy. Antimicrob Agents Chemother 40, 1577-1583.

Perfect, J. R., Rude, T. H., Penning, L. M. \& Johnston, S. A. (1992). Cryptococcus neoformans TRP1 gene by complementation in Saccharomyces cerevisiae. Gene 122, 213-217.
Perry, C. M. \& Balfour, J. A. (1999). Fomivirsen. Drugs 57, 375-380.

Persidis, A. (1999). Antisense therapeutics. Nat Biotechnol 17, 403-404.

Rosas, A. L., Nosanchuk, J. D., Feldmesser, M., Cox, G. M., McDade, H. M. \& Casadevall, A. (2000). Synthesis of polymerized melanin by Cryptococcus neoformans in rodents. Infect Immun 68, 2845-2853.

Salas, S. D., Bennett, J. E., Kwon-Chung, K. J., Perfect, J. R. \& Williamson, P. R. (1996). Effect of the laccase gene, CNLAC1, on virulence of Cryptococcus neoformans. J Exp Med 184, 377-386.

Tentler, S., Palas, J., Enderlin, C., Campbell, J., Taft, C., Miller, T. K., Wood, R. L. \& Selitrennikoff, C. P. (1997). Inhibition of Neurospora crassa growth by a glucan synthase- 1 antisense construct. Curr Microbiol 34, 303-308.

Wickes, B. L. \& Edman, J. C. (1995). The Cryptococcus neoformans $G A L 7$ gene and its use as an inducible promoter. Mol Microbiol 16, 1099-1109.

Received 4 May 2001; revised 5 September 2001; accepted 11 September 2001. 